\title{
PROPERTIES OF ABSOLUTE SUMMABILITY MATRICES
}

\section{J. A. FRIDY}

1. Introduction. Let $A$ denote a matrix summability method that maps the complex number sequence $x$ into the sequence $A x$ whose $n$th term is given by

$$
(A x)_{n}=\sum_{k \geq 1} a_{n k} x_{k} .
$$

If $A x$ is in $l^{1}$ whenever $x$ is in $l^{1}$, then $A$ is called an $l-l$ matrix. An $l-l$ matrix $A$ is said to be sum-preserving if for each $x$ in $l^{1}$,

$$
\sum_{n \geq 1}(A x)_{n}=\sum_{k \geq 1} x_{k}
$$

The inverse image of $l^{1}$ under $A$ is denoted by $l_{A}$.

In $[7$, p. 129] Steinhaus proved that if $A$ is a regular (i.e., limitpreserving) matrix, then there is a sequence of 0 's and 1's such that $A x$ is not convergent. It follows immediately that a regular matrix cannot sum every bounded sequence. (Cf. [6].) The principal result of this paper is the analogue of this theorem for $l$ - $l$ matrices.

The author is indebted to H. I. Brown for several helpful comments and observations.

2. The main theorem.

Theorem 1. If $A$ is a sum-preserving $l$-l matrix and $p>1$, then $l^{p} \Phi l_{A}$.

By using the characterization of sum-preserving $l-l$ matrices given in [5], we see that this theorem is an immediate corollary to the following assertion.

Theorem 2. If $p>1$ and $A$ is an l-l matrix such that

$$
\lim \sup _{k} \sum_{n \geq 1}\left|a_{n k}\right|>0
$$

then $l^{p} \Phi l_{A}$.

PRoof. We may assume that for each $n, \lim _{k} a_{n k}=0$, for otherwise the conclusion is trivial. Let $\epsilon$ be a positive number such that for infinitely many $k, \sum_{n \geqq 1}\left|a_{n k}\right| \geqq 2 \epsilon$. We now construct integer sequences $\kappa$ and $\nu$ in the following manner. 1969.

Presented to the Society, January 26, 1969; received by the editors March 28, 
Choose $\kappa(1)$ so that $\sum_{n \geqq 1}\left|a_{n, \kappa(1)}\right| \geqq 2 \epsilon$, then choose $\nu$ (1) such that

$$
\sum_{n=1}^{\nu(1)}\left|a_{n, \kappa(1)}\right|>\epsilon \text { and } \sum_{n>\nu(1)}\left|a_{n, \kappa(1)}\right|<2^{-1} \text {. }
$$

Having defined $\kappa(i)$ and $\nu(i)$ for $i<m$, we choose $\kappa(m)$ greater than $\kappa(m-1)$ such that

$$
\sum_{n=1}^{v(m-1)}\left|a_{n, \kappa(m)}\right|<2^{-m} \text { and } \sum_{n \geqq 1}\left|a_{n, \kappa(m)}\right| \geqq 2 \epsilon .
$$

Then choose $\nu(m)$ greater than $\nu(m-1)$ and satisfying

$$
\sum_{n=1+\nu(m-1)}^{\nu(m)}\left|a_{n, \kappa(m)}\right|>\epsilon \text { and } \sum_{n>\nu(m)}\left|a_{n, \kappa(m)}\right|<2^{-m} .
$$

If $k=\kappa(i)$ define $x_{k}=i^{-1}$, otherwise $x_{k}=0$; obviously $x$ is in $l^{p}$. Defining $\nu(0)=0$, we have

$$
\begin{aligned}
\sum_{n=1}^{\nu(N)}\left|(A v)_{n}\right| & =\sum_{m=1}^{N} \sum_{n=1+\nu(m-1)}^{\nu(m)}\left|\sum_{i \geqq 1} i^{-1} a_{n, \kappa(i)}\right| \\
& \geqq \sum_{m=1}^{N} \sum_{n=1+\nu(m-1)}^{\nu /(m)}\left\{\left|a_{n, \kappa(m)}\right| m^{-1}-\sum_{i \neq m}\left|a_{n, \kappa(i)}\right|\right\} \\
& >\sum_{m=1}^{N} \epsilon m^{-1}-\sum_{m=1}^{N} \sum_{n=1+\nu(m-1)}^{\nu(m)} \sum_{i \neq m}\left|a_{n, \kappa(i)}\right| .
\end{aligned}
$$

Since

$$
\sum_{m \geqq 1} \sum_{n=1+\nu(m-1)}^{\nu(m)} \sum_{i<m}\left|a_{n, \kappa(i)}\right|<\sum_{m \geqq 1} 2^{-m}
$$

and

$$
\sum_{m \geqq 1} \sum_{n=1+\nu(m-1)}^{\nu(m)} \sum_{i>m}\left|a_{n, \kappa(i)}\right|<\sum_{m \geqq 1} 2^{-m-1}
$$

it follows that $A x$ is not in $l^{1}$.

3. Further remarks and results about $l_{A}$. It is worthwhile noting that Theorem 2 gives a necessary condition for $A$ to map $l^{p}$ into $l^{1}$ : viz.,

$$
\lim _{k} \sum_{n \geq 1}\left|a_{n k}\right|=0
$$


However, this condition is not sufficient to imply that $l^{p} \subseteq l_{A}$, even for diagonal matrices; e.g., consider diag $\{1 / \log (n+1)\}$.

Property (2) does yield some information about the summability field $l_{A}$, but rather than limiting the size of $l_{A}$, (2) implies that $l_{A}$ cannot be too small. This statement is put in precise language in the next result, which is easily proved.

Proposition. If $A$ is a matrix such that

$$
\lim \inf _{k} \sum_{n \geqq 1}\left|a_{n k}\right|=0,
$$

then $l_{A} \Phi l^{p}$.

By an obvious modification of the proof of Theorem 2 we can show that a sum-preserving $l-l$ matrix cannot map every sequence of 0 's and 1's into $l^{1}$. This is precisely the $l-l$ analogue of Steinhaus' theorem. In [2] R. C. Buck used the Steinhaus theorem to prove a summability characterization of convergent sequences: viz., the bounded sequence $x$ is convergent if and only if there exists a regular matrix that sums every subsequence of $x$. In the light of the preceding remarks one might conjecture that members of $l^{1}$ could be characterized by the existence of a sum-preserving $l-l$ matrix for which $l_{\boldsymbol{A}}$ contains every subsequence. This, however, is false.

EXAMPLE. Let $A$ be the matrix mapping given by

$$
(A x)_{1}=x_{1}-x_{2} \text { and }(A x)_{n}=2 x_{n}-x_{2 n-1}-x_{2 n} \quad \text { if } n>1 .
$$

Then $A$ is clearly a sum-preserving $l-l$ matrix, but if $x$ is a constant sequence then $(A y)_{n} \equiv 0$ for every subsequence $y$ of $x$.

\section{REFERENCES}

1. R. P. Agnew, Summability of subsequences, Bull. Amer. Math. Soc. 50 (1944), 596-598. MR 6, 46.

2. R. C. Buck, A note on subsequences, Bull. Amer. Math. Soc. 49 (1943), 898-899. MR 5, 117.

3. J. A. Fridy, A note on absolute summability, Proc. Amer. Math. Soc. 20 (1969), 285-286. MR 38 \#1428.

4. G. H. Hardy, Divergent series, Clarendon Press, Oxford, 1949, MR 11, 25.

5. K. Knopp and G. G. Lorentz, Beiträge zur absoluten Limitierung, Arch. Math. 2 (1949), 10-16. MR 11, 346.

6. I. Schur, Über linear Transformationen in der Theorie die unendlich Reihen, J. Reine Angew Math. 151 (1921), 79-111.

7. H. Steinhaus, Some remarks on the generalization of limit, Prace Mat. Fiz. 22 (1911), 121-134.

Kent State University 